\title{
Electron transport chain dysfunction in neonatal pressure-overload hypertrophy precedes cardiomyocyte apoptosis independent of oxidative stress
}

\author{
Eric R. Griffiths, MD, Ingeborg Friehs, MD, Elisabeth Scherr, MD, Dimitrios Poutias, BS, \\ Francis X. McGowan, MD, and Pedro J. del Nido, MD
}

\begin{abstract}
Objectives: We have previously shown in a model of pressure-overload hypertrophy that there is increased cardiomyocyte apoptosis during the transition from peak hypertrophy to ventricular decompensation. Electron transport chain dysfunction is believed to play a role in this process through the production of excessive reactive oxygen species. In this study we sought to determine electron transport chain function in pressure-overload hypertrophy and the role of oxidative stress in myocyte apoptosis.
\end{abstract}

\begin{abstract}
Methods and Results: Neonatal rabbits underwent thoracic aortic banding at 10 days of age. Compensated hypertrophy ( 4 weeks of age), decompensated hypertrophy ( 6 weeks of age), and age-matched controls ( $n=4-8$ per group) as identified by serial echocardiography were studied. Electron transport chain complex activities were determined by spectophotometry in isolated mitochondria. Complex I was significantly decreased $(P=.005)$ at 4 weeks and further decreased at 6 weeks $(P=.001)$. Complex II was significantly decreased at both time points (4 weeks, $P=.003$; 6 weeks, $P=.009$ ). However, hyddrogen peroxide production, measured in isolated mitochondria by fluorescence spectroscopy, was significantly decreased at 4 weeks of age in banded animals compared with controls $(P=.038)$, and mitochondrial DNA oxidative damage (measurement of 8-hydroxydeoxyguanosine by enzyme-linked immunosorbent assay) was also significantly decreased at 4 weeks of age $(P=$ .031). Mitochondrial activated apoptosis was determined by Bax/Bcl-2 ratios (immunoblotting). Bax/Bcl-2 levels were significantly increased in banded animals at 6 weeks.
\end{abstract}

Conclusions: In pressure-overload hypertrophy, the transition from compensated left ventricular hypertrophy to failure and cardiomyocyte apoptosis is preceded by mitochondrial complex I and II dysfunction followed by an increase in $\mathrm{Bax} / \mathrm{Bcl}-2$ ratios. The mechanism of apoptosis initiation is independent of increased oxidative stress. (J Thorac Cardiovasc Surg 2010;139:1609-17)

Pressure-overload hypertrophy is the ventricular response to chronically increased systolic stress in an attempt to normalize wall stress and maintain contractility. Left ventricular hypertrophy $(\mathrm{LVH})$ is a risk factor for sudden death and progression to decompensated hypertrophy or heart failure and its associated morbidity and mortality. ${ }^{1}$ Cardiomyocyte loss through apoptosis is believed to play a role in the transition from compensated LVH to decompensated LVH. We have previously shown in a model of pressure-overload hypertrophy that there is increased cardiomyocyte loss

\footnotetext{
From the Departments of Cardiac Surgery and Anesthesiology, Perioperative and Pain, Children's Hospital Boston, Harvard Medical School, Boston, Mass.

Supported by grants from National Heart, Lung and Blood Institute HL-075430 (to I. Friehs) and HL-063095 (to P. J. del Nido), and HL-074734 and HL-0066186 (to F. X. McGowan). Dr Griffiths was supported by the Harvard-Longwood Research Training in Vascular Surgery: T32 HL 007734 to F. W. Logerfo.

Disclosures: None.

Received for publication Feb 4, 2009; revisions received July 3, 2009; accepted for publication Aug 9, 2009; available ahead of print Dec 28, 2009.

Address for reprints: Pedro J. del Nido, MD, Department of Cardiac Surgery, Children's Hospital Boston, 300 Longwood Ave, Boston, MA 02115 (E-mail: pedro. delnido@cardio.chboston.org).

$0022-5223 / \$ 36.00$

Copyright (c) 2010 by The American Association for Thoracic Surgery

doi:10.1016/j.jtcvs.2009.08.060
}

through apoptosis during the transition from peak hypertrophy to ventricular decompensation and dilation. ${ }^{2,3}$ No clear mechanism has been identified, but mitochondria present an attractive target for investigation because of their gatekeeper role in apoptosis, as well as mounting evidence for electron transport chain (ETC) dysfunction in various models of heart failure.

Oxidative phosphorylation occurs via the ETC located on the inner mitochondrial membrane. The ETC is composed of four electron transport protein complexes and the adenosine triphosphate (ATP) synthase that harnesses the proton motive force to generate ATP. Mitochondria isolated from failing myocardium have shown impaired capacity for respiration, various defects in ETC complexes, and decreased oxidative phosphorylation. ${ }^{4,5}$

A by-product of oxidative phosphorylation is the generation of reactive oxygen species (ROS). The single electron reduction of molecular oxygen at various sites in the ETC results in superoxide formation. ${ }^{6,7}$ Approximately $2 \%$ of all oxygen consumed in oxidative phosphorylation results in ROS. ${ }^{8}$ The superoxide anion generated at the ETC complexes is converted to hydrogen peroxide $\left(\mathrm{H}_{2} \mathrm{O}_{2}\right)$ by the enzyme manganese superoxide dismutase located in the 


$$
\begin{aligned}
& \text { Abbreviations and Acronyms } \\
& \begin{aligned}
\text { ATP } & =\text { adenosine triphosphate } \\
\mathrm{BSA} & =\text { bovine serum albumin } \\
\mathrm{ETC} & =\text { electron transport chain } \\
\mathrm{HIF}-1 \alpha & =\text { hypoxia-inducible factor- } 1 \alpha \\
\mathrm{H}_{2} \mathrm{O}_{2} & =\text { hydrogen peroxide } \\
\mathrm{KCN} & =\text { potassium cyanide } \\
\mathrm{LV} & =\text { left ventricle (ventricular) } \\
\mathrm{LVH} & =\text { left ventricular hypertrophy } \\
\text { 8-OHdG } & =\text { hydroxydeoxyguanosine } \\
\mathrm{ROS} & =\text { reactive oxygen species } \\
\text { TUNEL } & =\text { terminal deoxynucleotidyl transferase- } \\
& \text { mediated dUTP nick-end labeling }
\end{aligned}
\end{aligned}
$$

mitochondrial matrix. $\mathrm{H}_{2} \mathrm{O}_{2}$ can be further detoxified to $\mathrm{H}_{2} \mathrm{O}$ by other endogenous scavenging mechanisms such as catalase and glutathione peroxidase. There is evidence that low concentrations of superoxide can function "physiologically" as an intracellular messenger in various redoxsensitive signaling pathways including hypoxia-inducible factor- $1 \alpha$ (HIF-1 $\alpha)$ and phosphatidylinositol-3 kinase/ Akt. ${ }^{9,10}$ Overproduction of ROS and/or inadequate endogenous scavenging mechanisms can result in mitochondrial and cellular damage, ultimately leading to apoptosis. ${ }^{11}$

In ischemia-reperfusion injury, as well as in models of acute myocardial infarction, mitochondrial dysfunction leads to increased ROS generation and resultant peroxidation of lipids, proteins, and DNA. ${ }^{12}$ Mitochondrial peroxidative damage results in release of proapoptotic factors and initiation of apoptosis. However, in the pressure-loaded ventricle, the relationship and possible mechanism between mitochondrial dysfunction and apoptosis remain unclear. We hypothesize that the ETC impairment is the cause of mitochondrial dysfunction in hypertrophied myocardium and subsequent myocyte apoptosis. In this study, we sought to also determine whether ROS production plays a role in the mechanism of apoptosis.

\section{METHODS \\ LVH Model}

Pressure-overload hypertrophy was achieved by placing a silk ligature around the descending thoracic aorta of 10 day-old New Zealand White rabbits (Millbrook Farms, Amherst, Mass). The morphologic performance (heart weight and heart weight/body weight ratios) and the echocardiographic performance of this model over time have been previously described and validated in more detail by our group. ${ }^{2,13,14}$ Implanting a fixed constriction in an immature animal and allowing it to grow induced pressure-overload hypertrophy by 3 to 4 weeks of age in this model. The progression of LVH was determined by weekly transthoracic echocardiography. During these procedures, the animals remained unsedated to avoid the influences of anesthetics on the results. Transthoracic echocardiography was used to measure left ventricular (LV) wall thickness and cavity volume as previously described. ${ }^{13,15}$ The development of hypertrophy and progres- sion to failure were documented by LV mass/volume ratios as previously described. ${ }^{14}$ As a measure of cardiac performance, shortening fraction was determined with this formula: (diastolic diameter - systolic diameter)/diastolic diameter; it is expressed as a percentage.

Animals ( $n=4-8$ per group of banded and age-matched controls) were humanely killed with anesthetic overdose (ketamine/xylazine) and hearts were excised and placed on a Langendorff perfusion apparatus. After perfusion with Krebs-Henseleit solution for 5 minutes to wash out residual blood, the $\mathrm{LV}$ was frozen in liquid nitrogen and stored at $-80^{\circ} \mathrm{C}$ for future use.

\section{Isolation of Mitochondria}

Mitochondria were isolated from LV samples as previously described. ${ }^{16}$ In brief, frozen LV samples were pulverized in liquid nitrogen and suspended in isolation media containing $300 \mathrm{mmol} / \mathrm{L}$ sucrose, $10 \mathrm{mmol} / \mathrm{L}$ $\mathrm{K}^{+}$-HEPES buffer, $\mathrm{pH} 7.2$, and $1 \mathrm{mmol} / \mathrm{L} \mathrm{K}{ }^{+}$-ethyleneglutaminetetraacetic acid, $\mathrm{pH}$ 8.0. Samples were homogenized with glass grinding vessel for approximately 30 seconds. Nagarse $(0.2 \mathrm{mg} / \mathrm{mL})$ was added to the homogenate, which was incubated on ice for 10 minutes and then centrifuged for 5 minutes at $750 \mathrm{~g}$ at $4^{\circ} \mathrm{C}$. The supernatant was saved and $1 \mathrm{mg} / \mathrm{mL}$ bovine serum albumin (BSA) was added. The supernatant was then recentrifuged for 4 minutes at $750 \mathrm{~g}$ at $4^{\circ} \mathrm{C}$, and the pellet was discarded. The resulting supernatant was then centrifuged at $9000 \mathrm{~g}$ for 10 minutes at $4^{\circ} \mathrm{C}$. The mitochondrial pellet was then twice resuspended in ice-cold isolation media containing $1 \mathrm{mg} / \mathrm{mL}$ BSA and recentrifuged at $9000 \mathrm{~g}$ at $4^{\circ} \mathrm{C}$. The final pellet was suspended in 0.5 to $1 \mathrm{~mL}$ of isolation media (no BSA). Mitochondrial protein concentrations were determined by the bicinchoninic acid method.

\section{Immunoblotting}

Protein levels of representative subunits of complexes I and II, Bax, and Bcl2 were determined by immunoblotting. The total tissue protein extracts were separated by gel electrophoresis with sodium dodecylsulfate-polyacrylamide gel electrophoresis gels. Equal protein loading was confirmed by Coomassie blue staining of the gels. Proteins were electrophoretically transferred to nitrocellulose membranes, incubated in 5\% nonfat dry milk in TBST $(10 \mathrm{mmol} / \mathrm{L}$ Tris $\mathrm{HCl} \mathrm{pH} \mathrm{7.4,100} \mathrm{mmol/L} \mathrm{NaCl}$, and $0.1 \%$ Tween 20) for 1 hour and then incubated overnight with primary antibody against complex I (iron-sulfur protein 3 and ND6 protein from Invitrogen Corporation, Carlsbad, Calif), complex II (succinate dehydrogenase complex, subunit A [SDHA] flavoprotein from Invitrogen), Bax, and Bcl-2 (Millipore, Billerica, Mass). This was followed by incubation with horseradish peroxidase-conjugated secondary antibody with primary antibody at a dilution of 1:1000. The bound antibody was detected by enhanced chemiluminescence method according to the manufacturer's protocol (GE Healthcare Life Sciences, Waukesha, Wis). After exposure on film, quantitative protein analysis was conducted by laser densitometry. Data are expressed as arbitrary densitometry units.

\section{ETC Activity}

Mitochondrial ETC complex I, II, and III activities were measured by spectophotometry in isolated mitochondria as previously described. ${ }^{17}$ For complex I, mitochondria $(20 \mu \mathrm{g} / \mathrm{mL})$ underwent three cycles of freeze-thawing in hypotonic media $(25 \mathrm{mmol} / \mathrm{L}$ potassium phosphate and $5 \mathrm{mmol} / \mathrm{L}$ $\mathrm{MgCl}_{2}$ ) and were then added to buffer containing $25 \mathrm{mmol} / \mathrm{L} \mathrm{KH}_{2} \mathrm{PO}_{4}, 5$ $\mathrm{mmol} / \mathrm{L} \mathrm{MgCl}_{2}, 2 \mathrm{mmol} / \mathrm{L}$ potassium cyanide $(\mathrm{KCN}), 2.5 \mathrm{mg} / \mathrm{mL}$ BSA, 2 $\mu \mathrm{g} / \mathrm{mL}$ antimycin $\mathrm{A}$, and $0.13 \mathrm{mmol} / \mathrm{L}$ reduced nicotinamide adenine dinucleotide. The decrease in absorbance resulting from the oxidation of reduced nicotinamide adenine dinucleotide was measured at $345 \mathrm{~nm}$. Rotenone (2 $\mu \mathrm{g} / \mathrm{mL}$ ) was added and rotenone sensitive activity was recorded. Complex II activity was measured in mitochondria after three cycles of freeze-thawing in hypotonic media $(25 \mathrm{mmol} / \mathrm{L}$ potassium phosphate and $5 \mathrm{mmol} / \mathrm{L}$ $\mathrm{MgCl}_{2}$ ) and then preincubated in buffer containing $25 \mathrm{mmol} / \mathrm{L} \mathrm{KH}_{2} \mathrm{PO}_{4}$, $5 \mathrm{mmol} / \mathrm{L} \mathrm{MgCl}_{2}$, and $20 \mathrm{mmol} / \mathrm{L}$ succinate for 10 minutes at $30^{\circ} \mathrm{C}$ followed by addition of antimycin A ( $2 \mu \mathrm{g} / \mathrm{mL})$, rotenone $(2 \mu \mathrm{g} / \mathrm{mL}), \mathrm{KCN}(2 \mathrm{mmol} /$ 
$\mathrm{L})$, and dichlorophenolindophenol ( $50 \mu \mathrm{mol} / \mathrm{L})$. The reaction was catalyzed by addition of $65 \mu \mathrm{mol} / \mathrm{L}$ ubiquinone, and the reduction of dichlorophenolindophenol was measured at $600 \mathrm{~nm}$ for 2 to 3 minutes. Complex III activity was determined by measuring the increasing reduction of cytochrome $\mathrm{c}$ at $550 \mathrm{~nm}$. Mitochondria were added to buffer containing $25 \mathrm{mmol} / \mathrm{L}$ $\mathrm{KH}_{2} \mathrm{PO}_{4}, 5 \mathrm{mmol} / \mathrm{L} \mathrm{MgCl}, 2 \mathrm{mmol} / \mathrm{L} \mathrm{KCN}, 2 \mu \mathrm{g} / \mathrm{mL}$ rotenone, and 15 $\mu \mathrm{mol} / \mathrm{L}$ cytochrome $\mathrm{c}$ followed by the addition of ubiquinol $(35 \mu \mathrm{mol} / \mathrm{L})$. Complex IV activity was measured by the cytochrome c oxidase kit (CYTOCOX1) from Sigma-Aldrich (St Louis, Mo).

\section{Determination of ROS Production}

ROS production by mitochondria was determined by measuring the generation of $\mathrm{H}_{2} \mathrm{O}_{2}$ using fluorescence spectroscopy with Amplex Red Hydrogen Peroxide/Peroxidase Assay from Invitrogen in isolated mitochondria using $5 \mathrm{mmol} / \mathrm{L}$ glutamate and $5 \mathrm{mmol} / \mathrm{L}$ malate without the addition of adenosine diphosphate as the substrate for respiration. Mitochondria isolated from frozen tissue had $17 \%$ to $26 \%$ lower activity than mitochondria from fresh tissue, but the rate of the reaction was within an acceptable range. ROS-mediated DNA damage was assessed by measuring 8-hydroxydeoxyguanosine $(8-\mathrm{OHdG})$ in both nuclear and mitochondrial DNA by specific enzyme-linked immunosorbent assay (8-OHdG Check Ultrasensitive ELISA; BioVendor R\&D, Modrice, Czech Republic). Isolation of nuclear DNA was performed by digestion with proteinase $\mathrm{K}$ and then extracted in chloroform/isoamyl alcohol and phenol as previously described. ${ }^{18}$ Mitochondrial DNA was obtained by treating isolated mitochondria with proteinase $\mathrm{K}(10 \mathrm{mg} / \mathrm{mL})$ in sodium dodecylsulfate $(54 \mathrm{mg} / \mathrm{mL})$ followed by chloroform/isoamyl alcohol and phenol extraction and cold ethanol precipitation. ${ }^{18}$

\section{Determination of Apoptosis by TUNEL}

Cardiolmyocyte apoptosis was quantified by terminal deoxynucleotidyl transferase-mediated dUTP nick-end labeling (TUNEL) staining. LV muscle paraffin sections from the midsection of the heart were deparaffinized and then rehydrated with xylene and graded alcohol series. The sections were stained with the FragEL DNA fragmentation detection kit (EMD; Biosciences Inc, San Diego, Calif) per the manufacturer's instructions. In brief, the sections were incubated with terminal deoxynucleotidyl transferase and fluorescein-labeled dUTP. So that the cardiomyocytes could be identified, sections were incubated with mouse desmin monoclonal antibody (Sigma-Aldrich), followed by incubation with a secondary antimouse immunoreagent conjugated to the red-fluorescent Alexa-594 fluorophore at a concentration of 1:200 (Molecular Probes, Eugene Ore). Finally, so that all nuclei (nonapoptotic and apoptotic) could be identified, sections were stained with blue fluorescent DAPI nucleic acid stain (Molecular Probes). Coverslips were applied to the sections with fluorescent mounting medium (Dako Corporation, Carpinteria, Calif). Slides were visualized using an Axiovert 35 Microscope with a Nikon $10 \times$ objective, NA $=10$ $\times / 0.25$. Cardiomyocyte nuclei were determined by automatic counting with the computer software/image analyzer using the MetaMorph Imaging System software (Universal Imaging Corporation, West Chester, Pa). Apoptotic nuclei were identified manually to determine that only apoptotic cardiomyocyte nuclei were included. Data are expressed as apoptotic nuclei per 1000 nuclei.

\section{Animal Care}

All animals received humane care in compliance with the "Principles of Laboratory Animal Care" formulated by the National Society for Medical Research and the "Guide for the Care and Use of Laboratory Animals" prepared by the National Academy of Sciences and published by the National Institutes of Health (NIH Publication No. 86-23, revised 1996). The protocol was reviewed and approved by the Institutional Animal Care and Use Committee at Children's Hospital Boston.

\section{Statistical Analysis}

Data were analyzed with SPSS software package (version 15.0; SPSS Inc, Chicago, Ill) and are reported as mean \pm standard error of the mean. A 2-tailed unpaired Student $t$ test was used for comparison between groups: at 4 weeks (control versus banded) and at 6 weeks (control versus banded), if normality was passed, analysis of variance was used for analysis of the TUNEL staining where 3 groups were analyzed (control, 4 weeks banded, and 6 weeks banded.

\section{Statement of Responsibility}

The authors had full access to the data and take full responsibility for their integrity. All authors have read and agree to the manuscript as written.

\section{RESULTS \\ LVH Model}

After aortic banding, the animals had peak LVH at 4 to 5 weeks of age with a significantly increased mass/volume ratio (Figure 1). Cardiac performance, as measured by fractional shortening, was preserved at 4 weeks of age. By 6 weeks of age the mass/volume ratio had decreased significantly, indicating ventricular dilation, and was accompanied by contractile dysfunction evidenced by a decreased fractional shortening. These animals had no clinical signs of failure such as ascites or pleural effusions. Given these findings, animals were then studied at 4 weeks of age to evaluate compensated hypertrophy and at 6 weeks of age to evaluate decompensated hypertrophy.

\section{ETC Complex Activity and Protein Expression}

To identify the defect in oxidative phosphoryation, we measured the ETC complex activities by spectrophotometry in isolated mitochondria at 4 and 6 weeks of age. Complex I (Figure 2, A) showed significant impairment in the banded animals in hypertrophy. Complex II (Figure 2, B) also showed significant impairment. There was further impairment of both complexes with ventricular decompensation at 6 weeks of age. Complex III activity (Figure 2, C) was unchanged in both hypertrophy and decompensated hypertrophy. Complex IV activity (Figure 2, D) was slightly increased in the banded animals during LVH but normalized to control levels with ventricular dilation.

To determine whether the decrease in complex I and II activities was attributable to a decrease in complex subunit expression, we measured the protein levels of representative subunits by immunoblotting. Figure $3, A$, shows that the nuclear-encoded, $30-\mathrm{kDa}$ iron-sulfur protein 3 subunit of complex I was unchanged versus controls at both 4 and 6 weeks of age. Figure $3, B$, shows the $20-\mathrm{kDa}$, ND6 subunit of complex I, which is encoded by mitochondrial DNA, was unchanged at both time points as well. Figure 3, C, shows that the $70-\mathrm{kDa}$ flavoprotein subunit of complex II, which is the only ETC complex entirely encoded by nuclear DNA, was unchanged in both $\mathrm{LVH}$ and decompensated LVH. 

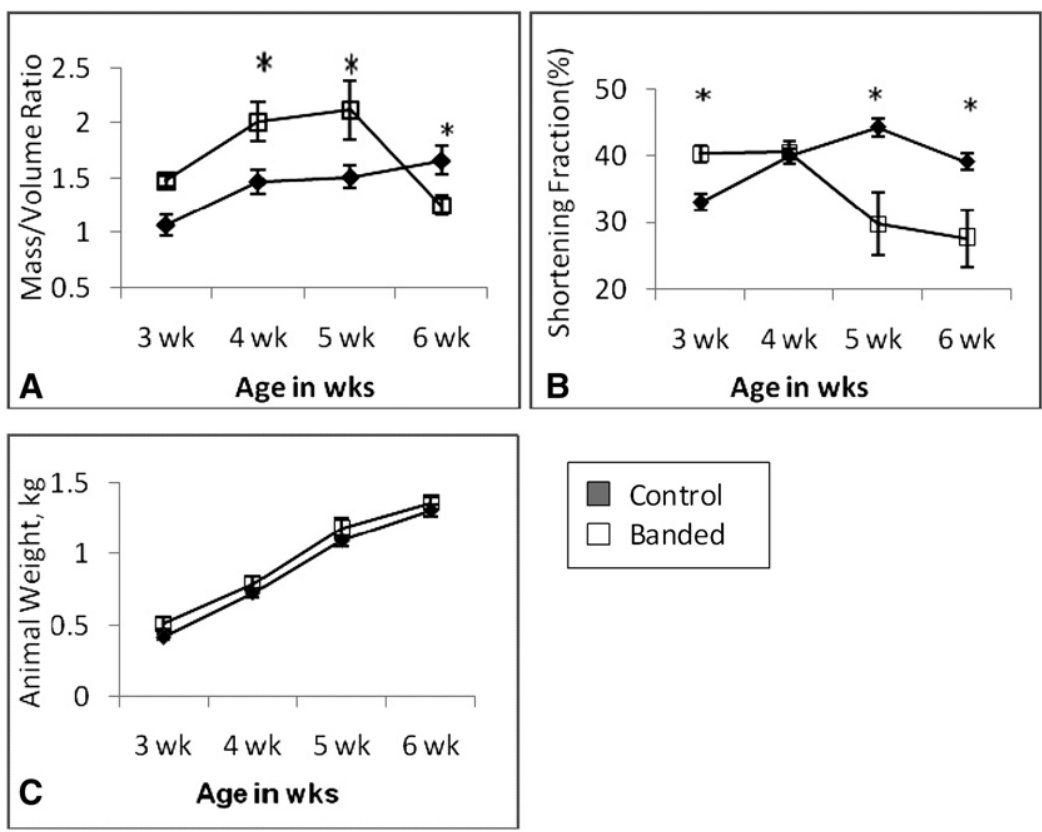

FIGURE 1. Transthoracic echocardiography showing development of LVH and later decompensation. A, Increase in mass/volume (M/V) ratio indicating that peak hypertrophy in the banded animals occurred at 4 to 5 weeks of age followed by ventricular dilatation at 6 weeks of age, evidenced by a decrease in $\mathrm{M} / \mathrm{V}$ ratio. B, Contractile performance was assessed by fractional shortening (FS). The FS was preserved during hypertrophy at 4 weeks of age and decreased as the ventricle dilated at 6 weeks $(n=6-11 ; * P \leq .05)$. C, There was no significant difference in animal body weights. Banded animals at 6 weeks of age had no clinical signs of heart failure such as ascites or pleural effusions.

\section{Determination of ROS Generation and Peroxidative Damage}

To determine whether complex I and II dysfunction results in altered ROS generation, we measured $\mathrm{H}_{2} \mathrm{O}_{2}$ production in isolated mitochondria using $5-\mathrm{mmol} / \mathrm{L}$ glutamate and $5-\mathrm{mmol} / \mathrm{L}$ malate as substrates for oxidative phosphorylation. Figure 4 shows that ROS production was significantly decreased in LVH at 4 weeks of age and

\section{Complex I}

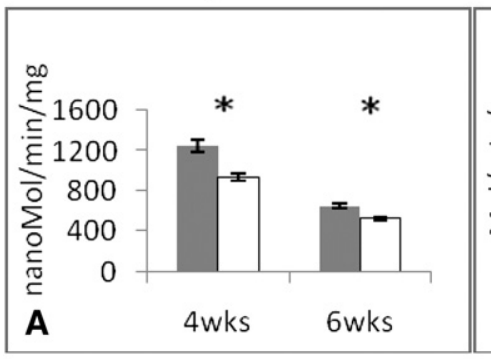

Complex III

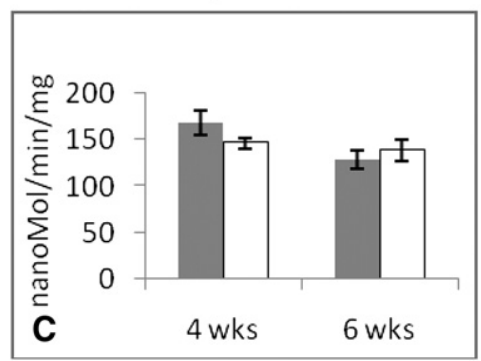

Complex II

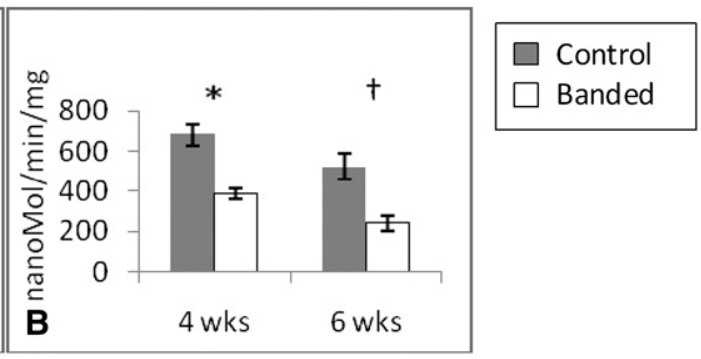

Complex IV

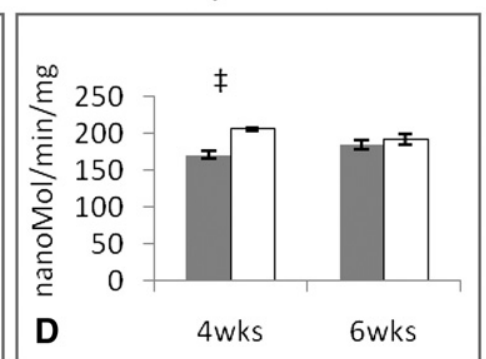

FIGURE 2. ETC complex activities measured by spectophotometry in isolated mitochondria from hypertrophied (4 weeks) and decompensated hypertrophy (6 weeks) LV myocardium. Complex I (A) and complex II (B) activities were significantly decreased at 4 weeks of age and further decreased significantly at 6 weeks of age. C, Complex III activity was unchanged. D, Complex IV activity was significantly increased at 4 weeks of age but normalizes at 6 weeks of age ( $\mathrm{n}=4 ; * P<.005, \dagger P=.009, \ddagger P=.03$ ). 


\section{Complex I- Nuclear endoded}
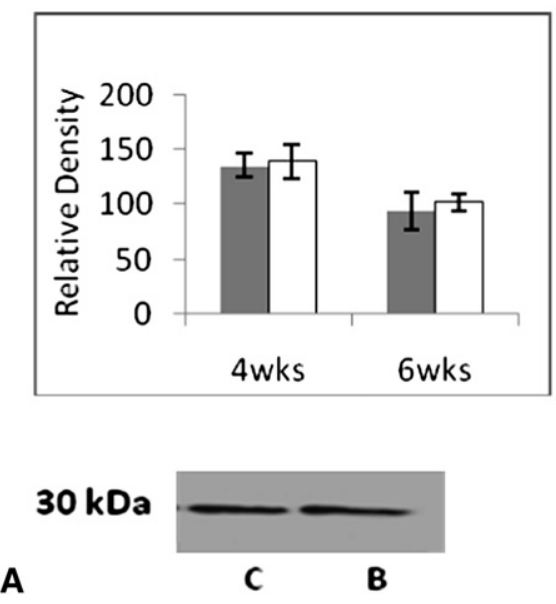

Complex II
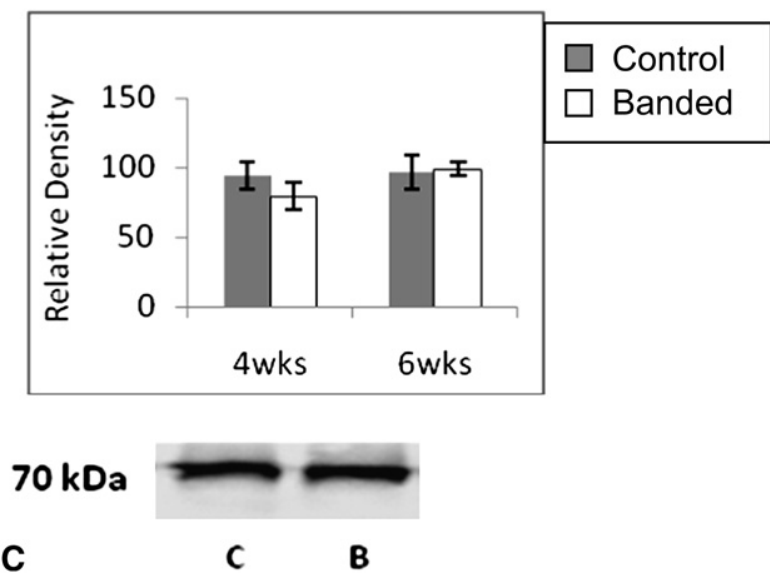

\section{Complex I-mtDNA encoded}

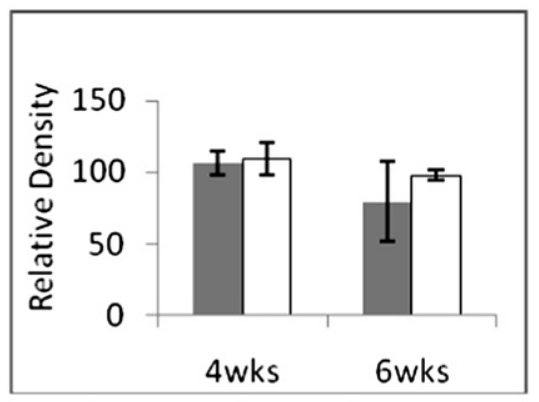

$20 \mathrm{kDa}$

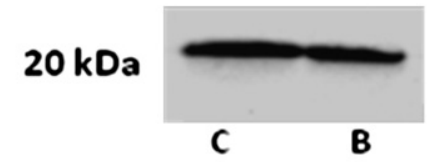

B

FIGURE 3. Western blot analysis of ETC complex subunit protein levels at 4 weeks and 6 weeks of age. Representative blots are shown. Nuclear encoded, 30-kDa iron-sulfur protein 3 (A) and the mitochondrial-encoded 20-kDa, ND6 subunits (B) of complex I are unchanged at both time points. C, The protein level of the complex II 70-kDa flavoprotein is unchanged change as well. Representative immunoblots are shown and bar graphs depict cumulative data, which are expressed are as means $\pm \operatorname{SEM}(n=4)$.

remained decreased in decompensated hypertrophy at 6 weeks of age. To confirm the decrease in ROS generation, we measured $8-\mathrm{OHdG}$, which is a marker for free radicalmediated DNA damage. There was no significant difference in the amount of oxidative damage in nuclear DNA (Figure 5, A). However, mitochondrial DNA contained significantly less oxidative damage in the hypertrophied LV at 4 weeks of age than controls (Figure 5, B). At 6 weeks of age, the amount of $8-\mathrm{OHdG}$ was decreased but not significantly in decompensated LV. These results are consistent with the findings of decreased $\mathrm{H}_{2} \mathrm{O}_{2}$ production in isolated mitochondria from hypertrophied LV.

\section{Activation of Apoptosis}

The presence of cardiomyocyte apoptosis was determined by TUNEL staining (Figure 5). There was a nonsignificant increase in cardiomyocyte apoptosis at 4 weeks of age compared with controls and a significant increase in banded animals at 6 weeks of age compared with controls. To investigate whether the mitochondrial dysfunction contributed to cardiomyocyte loss through activation of the intrinsic mitochondrial apoptotic pathway, we measured the proapoptotic and antiapoptotic proteins Bax and Bcl-2. In the decompensated LV at 6 weeks of age, there was a significant increase in the amount of Bax present (see Figure 6, $A$ ) and also in the ratio of $\mathrm{Bax} / \mathrm{Bcl}-2$ (Figure $6, B$ ), which is indicative of a proapoptotic state of the mitochondria that can lead to activation of the mitochondrial apoptosis pathway.

\section{DISCUSSION}

The major findings of the present study are as follows: (1) ETC dysfunction, specifically of complexes I and II, occurs 

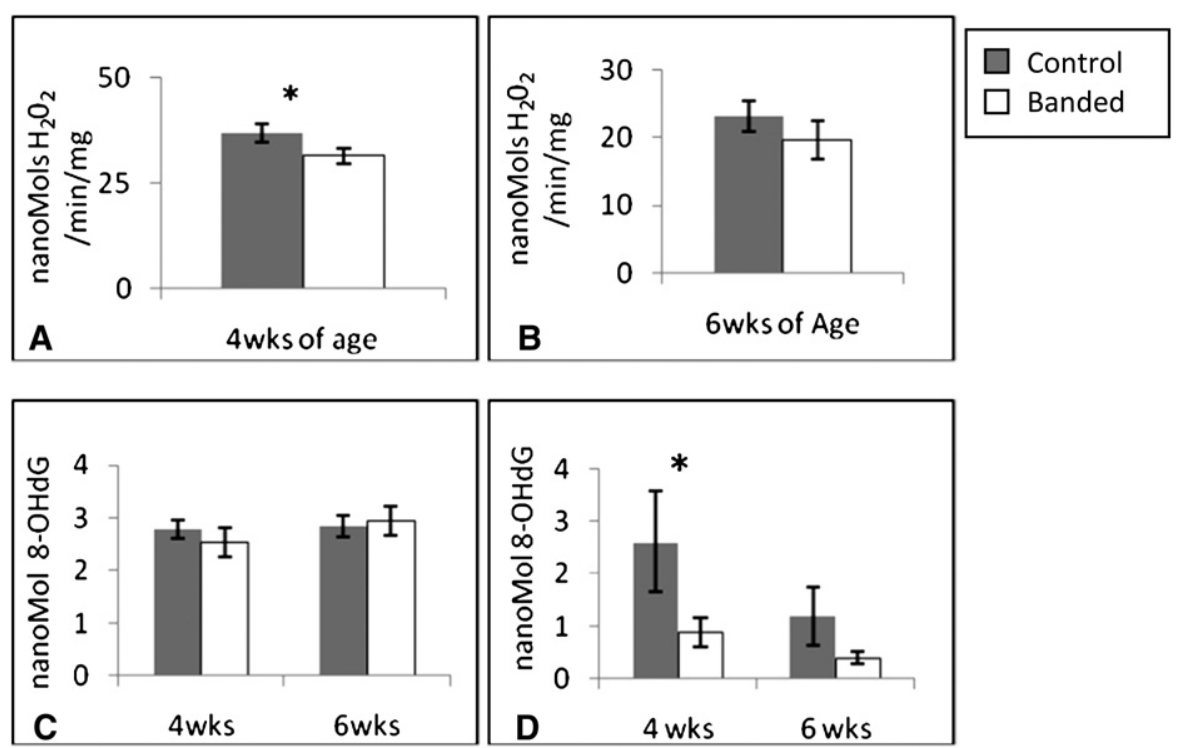

FIGURE 4. Determination of ROS generation and oxidative damage. Production of $\mathrm{H}_{2} \mathrm{O}_{2}$ was determined by fluorescence spectroscopy using the Amplex Red reaction in isolated mitochondria. A, Mitochondria at 4 weeks of age (hypertrophy) showed significantly decreased $\mathrm{H}_{2} \mathrm{O}_{2}$ production. B, Mitochondria at 6 weeks of age (decompenated hypertrophy) show decreased, but not significantly, production of $\mathrm{H}_{2} \mathrm{O}_{2}$. 8-OHdG, as a marker for oxidative DNA damage, was measured in digested nuclear and mitochondrial DNA. There was no difference in 8-OHdG levels in nuclear DNA (C) at either time point. In mitochondrial DNA (D), 8-OHdG levels were significantly decreased at 4 weeks of age (Data are expressed as means $\pm \mathrm{SEM} ; \mathrm{n}=4-6 ; * P \leq .05$ ).

in early hypertrophy and worsens with ventricular decompensation; (2) there is no change in protein content of complex I and II subunits; (3) ETC dysfunction is associated with decreased ROS generation; and (4) the mitochondrial dysfunction is associated with the activation of the mitochondrial apoptosis pathway, as indicated by $\mathrm{Bax} / \mathrm{Bcl}-2$ ratios. Collectively, these findings implicate ETC dysfunction as a potential cause of cardiomyocyte loss through apoptosis but through means other than excessive ROS production.

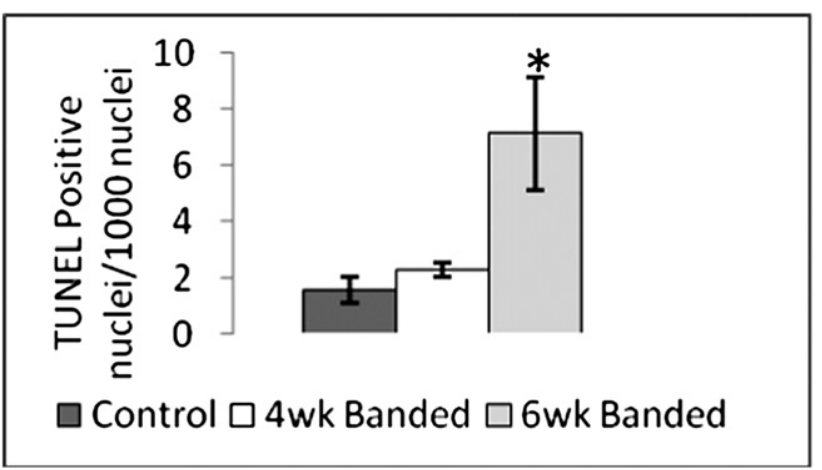

FIGURE 5. Western blot analysis of Bax and Bcl-2 protein levels at 6 weeks of age when cardiomyocyte apoptosis is known to be significantly increased. A, Significantly increased Bax protein levels in banded animals. B, Significantly increased Bax/Bcl-2 ratio. Representative immunoblots are shown and bar graphs depict cumulative data, which are expressed are as means \pm SEM $(\mathrm{n}=4 ; * P \leq .05)$.
Our findings in a model of a pressure-loaded ventricle show that complex I and II dysfunction precedes the onset of contractile dysfunction and ventricular dilatation. This indicates that initially the impairment is not sufficient to inhibit the production of adequate amounts of ATP for basal contractile function. The impairment could explain the reported finding of impaired ATP production at high work states in hypertrophied myocardium. ${ }^{19}$ Worsening complex dysfunction coincides with the onset of ventricular dilation and worsening contractile function. These data are consistent with the decreased amounts of ATP in failing myocardium reported in multiple models of heart failure. ${ }^{20}$ It must be noted that the impairment of ETC complex activities causing decreased oxidative phosphorylation has not been firmly established in heart failure and requires further investigation.

There was a significant decrease in activity in both complexes I and II in control animals from 4 weeks to 6 weeks of age. Because little is known about mitochondrial changes in the neonatal period, the use of a developing animal may confound the results seen in the control group, that is, decreases in activity of complexes I and II may occur with maturation of the animal. Despite the decreases in the activities of complexes I and II in the control animals, banded animals had significantly worse complex activities.

The decrease in complex activity is not attributable to a decrease in complex subunit proteins, as we found that representative subunits from complexes I and II were not decreased. Complex I is composed of 45 proteins, 7 of which are encoded by the mitochondrial genome. Complex II is the 


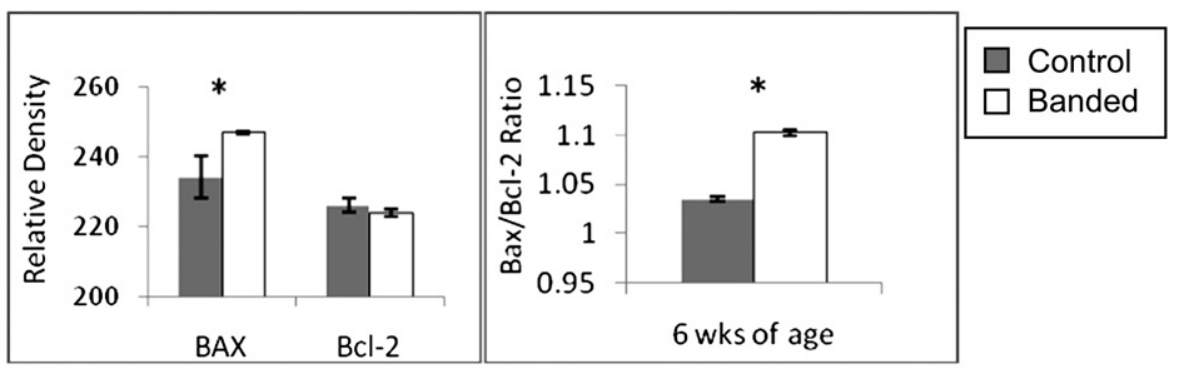

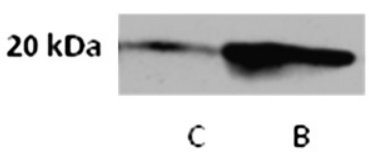

A

Bax
$25 \mathrm{kDa}$

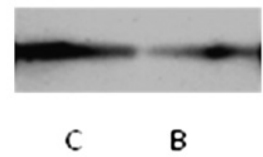

B

$\mathrm{BCl}-2$

FIGURE 6. Summarized results of TUNEL staining for cardiomyocyte apoptosis at 4 weeks and 6 weeks of age in aortic banded and control animals. There was a nonsignificant increase in cardiomyocyte apoptosis at 4 weeks of age compared with controls and a significant increase in banded animals at 6 weeks of age compared with controls. Results are expressed as means $\pm \mathrm{SD}(\mathrm{n}=3)$ of TUNEL-positive cardiomyocyte nuclei per 1000 nuclei. $* P=.025$.

only complex entirely encoded by nuclear DNA and is composed of 7 subunits. The subunits we elected to quantify have been reported as key subunits of each impaired complex. The 30-kDA, nuclear-encoded, iron-sulfur protein 3 subunit and mitochondrial-encoded ND6 subunit of complex I are both a part of the core electron transfer apparatus that is most likely to affect complex function if decreased in quantity. $^{21,22}$ The succinate dehydrogenase complex subunit A of complex II is a $70-\mathrm{kDa}$ flavoprotein involved in the electron transfer process. $^{23}$ Other possibilities exist that could explain the decreased function. Improper assembly of complexes by their constituent subunits or even posttranslation modifications could lead to impaired functioning as well. $^{24}$ Differential expression of ETC subunit isoforms that alters their activities has been described in yeast under hypoxic conditions, raising the possibility that isoform switching may occur as a result of the metabolic stress involved in the chronically pressure-loaded ventricle. ${ }^{25}$

The mechanism of impairment of ETC complex activity is not well understood. Recent studies have focused on possible posttranslational modifications that might function to regulate oxidative phosphorylation. Possible mechanisms include reversible processes such as phosphorylation, acetylation, palmytoylation, and glycosylation, and/or irreversible processes involving cleavage or degradation of the subunits. Many of these posttranslational modifications have been identified in complex I but remain under investigation. $^{26,27}$

We found that the impairment of complex I and II activity resulted in decreased production of $\mathrm{H}_{2} \mathrm{O}_{2}$ in isolated mitochondria from hypertrophied myocardium compared with age-matched controls. The decreased production of mitochondrial ROS was confirmed by the decreased amounts of $8-\mathrm{OHdG}$, a marker of ROS-induced DNA damage in mitochondrial DNA. Because of its proximity to the ETC, the lack of negatively charged histone proteins, and the less efficient DNA repair mechanisms, mitochondrial DNA is particularly susceptible to ROS-mediated damage. Complex I and II impairment decreases production of ROS by limiting the flux through the ETC. Inasmuch as ROS is a by-product of oxidative phosphorylation, a decrease in oxidative phosphorylation results in a proportional decrease in ROS generation.

The lack of nuclear DNA damage in hypertrophy and decompensated hypertrophy indicates that the ROS generated from all sources are sufficiently detoxified, preventing nuclear DNA damage. This is an important finding because other sources of ROS are reportedly active in the hypertrophied LV. The cytosolic reduced nicotinamide adenine dinucleotide phosphate oxidase enzyme has been found to play a role in LVH through ROS-dependent activation of mitogen-activated protein kinase pathways in angiotensin II and endothelin-induced models of hypertrophy. ${ }^{7,28}$ Elevated levels of ROS generated in a reduced nicotinamide adenine dinucleotide phosphate oxidase may be sufficient to induce hypertrophic signaling but insufficient to incur oxidative damage.

Our findings of decreased oxidative stress in pressureoverload hypertrophy are in contrast to the documented role ROS play in heart failure models from ischemia-reperfusion or acute myocardial infarction. In our model, the aortic band is placed in a nonconstricting fashion such that the pressure load gradually develops as do the structural and metabolic changes seen in LVH. This contrasts significantly with the models of ischemia-reperfusion and acute myocardial infarction, where a relatively healthy ventricle sustains an acute insult that leads to rapid development of multiple metabolic and structural abnormalities including 
cardiomyocyte apoptosis. However, the mechanism by which apoptosis is induced likely varies between the different models. Elevated ROS induce lipid and protein peroxidation in the inner mitochondrium, which in sufficient quantity can promote release of cytochrome $\mathrm{c}$ into the cytosol and activate apoptosis. Therefore, efforts in these models aim to decrease ROS generation or peroxidative damage through free radical scavenging. ${ }^{12}$

Our findings in a pressure-loaded ventricle show ETC impairment precedes the onset of cardiomyocyte apoptosis, which was found to be significantly increased at 6 weeks of age. ${ }^{3}$ We document the activation of the mitochondrial apoptotic pathway through an increase in the Bax/Bcl-2 ratio. Homodimerazation of Bax and incorporation into the outer mitochondrial membranes (which is prevented by $\mathrm{Bcl}-2)$ results in loss of mitochondrial membrane potential and leakage of proapoptotic proteins into the cytosol and ultimately activating downstream effector caspases. We demonstrated increased levels of Bax in decompensated hypertrophy hearts as well as an increased $\mathrm{Bax} / \mathrm{Bcl}-2$ ratio independent of increased oxidative stress. The increased $\mathrm{Bax} / \mathrm{Bcl}-2$ ratio at 6 weeks of age corresponds to the time when cardiomyocyte apoptosis is significantly increased in our model. ${ }^{3}$ The mechanism by which Bax levels are increased is not clear. However, increased oxidative stress does not appear to play a role. An important contributor may be the lack of appropriate signaling through the HIF$1 \alpha$ pathway, which is a key regulator of cell survival. ${ }^{29,30}$ $\mathrm{We}^{31}$ have previously found in our model that $\mathrm{HIF}-1 \alpha$ fails to translocate into the nucleus in hypertrophied hearts when subjected to hypoxia, indicating a defect in HIF- $1 \alpha$ signaling. Key to the nuclear translocation of HIF- $1 \alpha$ is stabilization by ROS, preventing its degradation. If decreased ROS production limits the stabilization and subsequent translocation of HIF- $1 \alpha$, then the compensatory response would be inadequate to mitigate the effects of hypoxia or cellular stress.

ETC dysfunction with a resultant decrease in ROS production is a key finding in the pathogenesis of LVH and its progression to failure. The magnitude of its contribution to cardiomyocyte apoptosis, either through impaired oxidative phosphorylation and energy production or through altered ROS metabolism, requires further study. To better define the cause of ETC complex dysfunction, it will be necessary to take a proteomics approach to assess complex protein composition and posttranslational modifications. Understanding the specific mechanisms of the ETC dysfunction is essential to design appropriate interventions to alter the progression of hypertrophy and development of cardiac failure.

Special thanks to Dr Moritz Wyler von Ballmoos and Dr Huamei He for their technical assistance.

\section{References}

1. Frey N, Olson EN. Cardiac hypertrophy: the good, the bad, and the ugly. Ann Rev Physiol. 2003;65:45-79.

2. Choi YH, Cowan DB, Moran AM, Colan SD, Stamm C, Takeuchi K, et al. Myocyte apoptosis occurs early during the development of pressure-overload hypertrophy in infant myocardium. J Thorac Cardiovasc Surg. 2009;137:1356-62.

3. Friehs I, Barillas R, Vasilyev NV, Roy N, McGowan FX, del Nido PJ. Vascular endothelial growth factor prevents apoptosis and preserves contractile function in hypertrophied infant heart. Circulation. 2006;114(1 Suppl):I290-5.

4. Stanley WC, Recchia FA, Lopaschuk GD. Myocardial substrate metabolism in the normal and failing heart. Physiol Rev. 2005;85:1093-129.

5. Beer M, Seyfarth T, Sandstede J, Landschutz W, Lipke C, Kostler H, et al. Absolute concentrations of high-energy phosphate metabolites in normal, hypertrophied, and failing human myocardium measured noninvasively with (31)PSLOOP magnetic resonance spectroscopy. J Am Coll Cardiol. 2002;40:1267-74.

6. Watanabe N, Zmijewski JW, Takabe W, Umezu-Goto M, Le Goffe C, Sekine A, et al. Activation of mitogen-activated protein kinases by lysophosphatidylcholine-induced mitochondrial reactive oxygen species generation in endothelial cells. Am J Pathol. 2006;168:1737-48.

7. Tsutsui H, Ide T, Kinugawa S. Mitochondrial oxidative stress, DNA damage, and heart failure. Antioxid Redox Signaling. 2006;8:1737-44.

8. Lee HC, Wei YH. Role of mitochondria in human aging. J Biomed Sci. 1997;4: 319-26.

9. Sugden PH, Clerk A. Oxidative stress and growth-regulating intracellular signaling pathways in cardiac myocytes. Antioxid Redox Signaling. 2006;8:2111-24.

10. Tu VC, Bahl JJ, Chen QM. Signals of oxidant-induced cardiomyocyte hypertrophy: key activation of p70 S6 kinase-1 and phosphoinositide 3-kinase. J Pharmacol Expl Ther. 2002;300:1101-10.

11. Kumar D, Jugdutt BI. Apoptosis and oxidants in the heart. J Lab Clin Med. 2003; 142:288-97.

12. Chen Q, Moghaddas S, Hoppel CL, Lesnefsky EJ. Ischemic defects in the electron transport chain increase the production of reactive oxygen species from isolated rat heart mitochondria. Am J Physiol Cell Physiol. 2008;294:C460-6.

13. Friehs I, Cao-Danh H, Nathan M, McGowan FX, del Nido PJ. Impaired insulinsignaling in hypertrophied hearts contributes to ischemic injury. Biochem Biophys Res Commun. 2005;331:15-22.

14. Moran AM, Friehs I, Takeuchi K, Stamm C, Hammer PE, McGowan FX, et al. Noninvasive serial evaluation of myocardial mechanics in pressure overload hypertrophy of rabbit myocardium. Herz. 2003;28:52-62.

15. Friehs I, Moran AM, Stamm C, Choi YH, Cowan DB, McGowan FX, et al. Promoting angiogenesis protects severely hypertrophied hearts from ischemic injury. Ann Thorac Surg. 2004;77:2004-10; discussion 2011.

16. Rousou AJ, Ericsson M, Federman M, Levitsky S, McCully JD. Opening of mitochondrial KATP channels enhances cardioprotection through the modulation of mitochondrial matrix volume, calcium accumulation, and respiration. Am J Physiol. 2004;287:H1967-76.

17. Birch-Machin MA, Briggs HL, Saborido AA, Bindoff LA, Turnbull DM. An evaluation of the measurement of the activities of complexes I-IV in the respiratory chain of human skeletal muscle mitochondria. Biochem Med Metab Biol. 1994; 51:35-42.

18. Ausubel FM, Brent R, Kingston RE, Moore DD, Seidman JG, Smith JA, et al., eds. Short protocols in molecular biology. 5th ed. Hoboken: Wiley; 2002.

19. Bache RJ, Zhang J, Murakami Y, Zhang Y, Cho YK, Merkle H, et al. Myocardial oxygenation at high workstates in hearts with left ventricular hypertrophy. Cardiovasc Res. 1999;42:616-26.

20. Ingwall JS. Energy metabolism in heart failure and remodelling. Cardiovasc Res. 2009;81:412-9.

21. Porcelli AM, Angelin A, Ghelli A, Mariani E, Martinuzzi A, Carelli V, et al. Respiratory complex I dysfunction due to mitochondrial DNA mutations shifts the voltage threshold for opening of the permeability transition pore toward resting levels. J Biol Chem. 2009;284:2045-52.

22. Hirst J, Carroll J, Fearnley IM, Shannon RJ, Walker JE. The nuclear encoded subunits of complex I from bovine heart mitochondria. Biochim Biophys Acta. 2003; 1604:135-50.

23. Rustin P, Rotig A. Inborn errors of complex II-unusual human mitochondrial diseases. Biochim Biophys Acta. 2002;1553:117-22.

24. Fontanesi F, Soto IC, Barrientos A. Cytochrome c oxidase biogenesis: new levels of regulation. IUBMB Life. 2008;60:557-68.

25. Fukuda R, Zhang H, Kim JW, Shimoda L, Dang CV, Semenza GL. HIF-1 regulates cytochrome oxidase subunits to optimize efficiency of respiration in hypoxic cells. Cell. 2007;129:111-22. 
26. Balaban RS. Domestication of the cardiac mitochondrion for energy conversion. J Mol Cell Cardiol. 2009;46:832-41.

27. Kim SC, Sprung R, Chen Y, Xu Y, Ball H, Pei J, et al. Substrate and functional diversity of lysine acetylation revealed by a proteomics survey. Mol Cell. 2006; 23:607-18.

28. Li JM, Gall NP, Grieve DJ, Chen M, Shah AM. Activation of NADPH oxidase during progression of cardiac hypertrophy to failure. Hypertension. 2002;40: 477-84.
29. Loor G, Schumacker PT. Role of hypoxia-inducible factor in cell survival during myocardial ischemia-reperfusion. Cell Death Differ. 2008;15:686-90.

30. Kido M, Du L, Sullivan CC, Li X, Deutsch R, Jamieson SW, et al. Hypoxia-inducible factor 1-alpha reduces infarction and attenuates progression of cardiac dysfunction after myocardial infarction in the mouse. J Am Coll Cardiol. 2005;46:21 16-24.

31. Friehs I, Barillas R, Roy N, Vasilyev NV, Martinez JF, McGowan FX, et al. Deferoxamine mediated activation of hypoxia inducible factor- $1 \alpha(\mathrm{HIF}-1 \alpha)$ upregulates target genes for protection of hypertrophied myocardium. Circulation. 2005;112(17). II-350. 